\title{
Introduction to the special volume on constraint programming, artificial intelligence, and operations research
}

\author{
J. Christopher Beck • Barbara M. Smith
}

Published online: 11 October 2008

(C) Springer Science+Business Media, LLC 2008

The Third International Conference on Integration of Artificial Intelligence (AI) and Operations Research (OR) Techniques in Constraint Programming (CP) for Combinatorial Optimization Problems (CPAIOR 2006) was held in Cork, Ireland, from May 31-June 2, 2006.

The conference received 67 submissions. After a full reviewing process, 20 papers were chosen for presentation at the conference and publication in the proceedings. Of these 20 , and after a second full refereeing process, seven papers have been selected to appear here in significantly extended form.

The goal of the CPAIOR conference series is to provide a forum for researchers to present approaches that highlight the integration of $\mathrm{CP}, \mathrm{AI}$, and $\mathrm{OR}$ techniques as well as to encourage cross-fertilization between traditionally separate communities that have a significant overlap in terms of research approaches, algorithms, and problems of interest.

The papers in this Special Volume reflect the diversity and range of the conference. At one extreme, the problem of representing and reasoning about preferences in concert with constraints is a problem that has mostly arisen in the AI community. In their paper, Wallace $\&$ Wilson show how a lexicographic preference representation can be used to express various kinds of preference information and how the representation can be integrated with constraint programming. At the other extreme, Cadoli \& Patrizi address the problem of separability of sub-problems in a Benders decomposition framework. Such decomposition methods, traditionally seen as OR techniques, have received significant interest from the CP community over the past five years.

Hybrid approaches that combine algorithms and approaches from different domains are of particular interest to the CPAIOR community. Two papers here investigate hybrid ap-

J.C. Beck (凶)

Department of Mechanical and Industrial Engineering, University of Toronto, 5 King's College Rd., Toronto, ON M5S 3G8, Canada

e-mail: jcb@mie.utoronto.ca

B.M. Smith

School of Computing, University of Leeds, Leeds LS2 9JT, UK

e-mail: bms@comp.leeds.ac.uk 
proaches to challenging combinatorial problems. Rasmussen \& Trick define the timetable constrained distance minimization problem, based on real-world experience with sports scheduling. The problem is addressed with a number of approaches including a hybrid integer programming/constraint programming approach and a branch-and-price algorithm that also employs both integer programming and constraint programming. Gabteni \& Gronkvist address the tail assignment problem in aircraft scheduling where specific aircraft (called "tails" due to unique tail numbers) must be assigned to specific trip legs subject to operational constraints and the desire to minimize an objective function. A hybrid approach using constraint programming within a column generation framework is proposed and extensively evaluated.

One important topic that has been studied widely in both AI and OR is that of representing and reasoning about uncertain and stochastic problems. Tarim et al. propose a novel framework for representing and reasoning about uncertainty within constraint programming in order to find reliable solutions. The approach, called event-driven probabilistic constraint programming, allows richer modeling of probabilistic constraints and dependencies amongst them to define a more realistic problem model. Van Hentenryck et al. study on-line reservation systems where commitments (i.e., reservations) must be made for customers, without knowledge of future customers or potential cancellations by existing customers. The paper shows how mathematical programming and dynamic programming can be used within an on-line stochastic framework to form a constant sub-optimality approximation.

A fundamental strength of constraint programming, and an often-exploited approach to incorporation of OR algorithms, is the use of global constraints. A global constraint represents a generic pattern of relationships among variables that is seen in a variety of problems and for which an efficient inference algorithm can often be developed. Such constraints play a significant role in both the modeling and solving of CP problems. Beldiceanu et al. present constraint inference algorithms for two forest constraints: the resource-forest where every vertex must be included in a tree that itself includes at least one "resource" vertex (e.g., each workstation must be connected to one of the printers) and the proper-forest constraint which states that the vertices must be covered by a specific number of proper trees.

Finally, we would like to thank the reviewers of the papers in this Special Volume. Their dedicated volunteer work in carefully reading and commenting on the papers has had a significant impact on the quality of the papers in this issue. 